\title{
The Molecular Immunology of Mucositis: Implications for Evidence-Based Research in Alternative and Complementary Palliative Treatments
}

\section{Francesco Chiappelli}

\author{
Division of Oral Biology and Medicine, UCLA School of Dentistry, Los Angeles, CA 90095-1668, USA
}

\begin{abstract}
The terms 'mucositis' and 'stomatitis' are often used interchangeably. Mucositis, however, pertains to pharyngeal-esophago-gastrointestinal inflammation that manifests as red, burn-like sores or ulcerations throughout the mouth. Stomatitis is an inflammation of the oral tissues proper, which can present with or without sores, and is made worse by poor dental hygiene. Mucositis is observed in a variety of immunosuppressed patients, but is most often consequential to cancer therapy. It appears as early as the third day of intervention, and is usually established by Day 7 of treatment. Mucositis increases mortality and morbidity and contributes to rising health care costs. The precise immune components involved in the etiology of mucositis are unclear, but evidence-based research (EBR) data has shown that applications of granulocyte-macrophage-colony stimulating factor prevent the onset or the exacerbation of oropharyngeal mucositis. The molecular implications of this observation are discussed from the perspective of future developments of complementary and alternative treatments for this condition. It must be emphasized that this article is meant to be neither a review on mucositis and the various treatments for it, nor a discussion paper on its underlying molecular immunology. It is a statement of the implications of EBR for CAM-based interventions for mucositis. It explores and discusses the specific domain of molecular immunology in the context of mucositis and its direct implications for EBR research in CAM-based treatments for mucositis.
\end{abstract}

Keywords: Mucositis - evidence-based research - molecular immunology - GM-CSF

\section{Mucositis}

The terms 'mucositis' and 'stomatitis' are often used interchangeably. Mucositis, however, pertains to pharyngealesophago-gastrointestinal inflammation that manifests as red, burn-like sore or ulcerations throughout the mouth. Stomatitis is an inflammation of the oral tissues proper, which can present with or without sores, and is made worse by poor dental hygiene.

Oropharyngeal mucositis is a common and treatmentlimiting side effects of immune suppression, such as those

For reprints and all correspondence: Francesco Chiappelli, PhD, Division of Oral Biology and Medicine, UCLA School of Dentistry, Los Angeles, CA 90095-1668, USA. Tel: +1-310-794-6625; Fax: +1-310-794-7109; E-mail: Chiappelli@dent.ucla.edu directed by the cytotoxic action of cancer therapy. It is an acute oral mucosal inflammatory reaction secondary to cell death of the basal cell lining of the oral mucosa. Oral microorganisms play an important role in aggravating the pathology of the impaired epithelium. Smoking, and alcohol use and abuse, and psycho-emotional stress are also relevant factors. Clinically observed atrophy (tissue damage) and telangiectasis (blood vessel, spider-like red spots) of the mucosa increase the risk for pain and/or necrosis. Mucositis leads to systemic immune suppression, and to an increased risk of local and generalized infection, opportunistic infections and mortality due to sepsis.

Mucositis is common among oncology patients. Whereas early estimates proposed that mucositis occurred in over $40 \%$ of patients who receive cancer chemotherapy or irradiation (1), more recent data indicate that oral and gastrointestinal mucositis can affect up to $100 \%$ of patients undergoing

(C) The Author (2005). Published by Oxford University Press. All rights reserved.

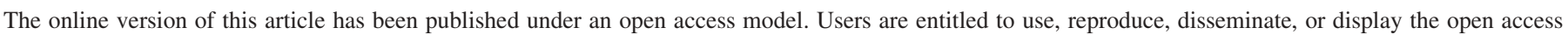

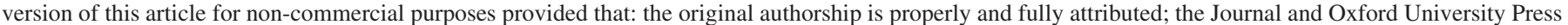

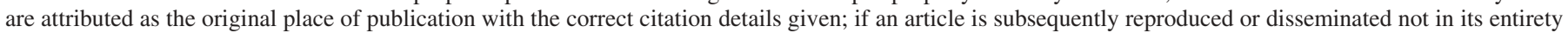
but only in part or as a derivative work this must be clearly indicated. For commercial re-use, please contact journals.permissions@oxfordjournals.org 
high-dose chemotherapy and hematopoietic stem cell transplantation, $80 \%$ of patients with malignancies of the head and neck receiving radiotherapy, and over $50 \%$ of patients receiving chemotherapy (2).

The severity of mucositis depends from the type of cancer, the schedule and nature of the chemotherapy intervention, the area irradiated and the amount of radiation given. In the case of radiation therapy, if it is delivered at a rate equivalent to the ability of the oral mucosa to regenerate, then only mild mucositis occurs. Chemotherapy treatment given by continuous infusion or frequent, repetitive, intermittent schedules are more likely to cause mucositis than the same drugs given by a single short infusion. Severe oral mucositis can lead to the need to interrupt or discontinue the cancer therapy protocol, thus hindering cure of the primary disease. Impairments of local oral immunity, and consequential infection are common manifestations of mucositis, and appropriate diagnosis and antimicrobial treatment interventions must be considered for both fungal and bacterial organisms. Viral infections are rarely a complication of mucositis (2-4).

A five-step model of mucositis has been proposed (4) to help explain the clinical features of the condition. The five phases of oropharyngeal mucositis are:

(i) initiation;

(ii) upregulation and message generation;

(iii) signaling and amplification;

(iv) ulceration; and

(v) healing.

These phases may be observed to occur simultaneously and recur cyclically in any given patient. The model offers a rational approach for understanding the fundamental pathobiology and for targeting various components of the pathologic process for research into therapeutic interventions (4).

The molecular and cellular pathways that lead to mucositis are unclear. Research suggests that the condition represents the culmination of a dynamic sequence of three distinct events in the oropharyngeal mucosa:

(i) keratinocyte toxicity and death;

(ii) impaired mucosal immune surveillance; and

(iii) significant alterations in oral flora (5).

These events can lead to ulcerative lesions in the oropharynx that can be so painful as to restrict oral intake of food and liquids. These lesions can also act as sites of secondary infection and portals of entry for the endogenous flora in the stoma (1).

\section{Palliative and Therapeutic Treatment}

Oropharyngeal mucositis manifests as inflammation, which can be severe and leading to lesions and ulcerations of the mucosa, and which can significantly impair the daily functioning and quality of life of the patients. Current care of patients with mucositis is essentially palliative and includes appropriate oral hygiene, non-irritating diet and oral care products, topical mouth rinses, topical anesthetics and opioid analgesics. Topical anesthetics (Viscous Xylocaine ${ }^{\circledR}$ ) have been reported to be of some value, unless the pain requires systemic analgesic drugs. Despite our understanding that the loss of rapidly dividing epithelial progenitor cells triggers the onset of the disorder, the severity and duration of the condition are determined largely by changes in local immunity, which lead to changes in oral flora. The complexity of the pathobiological processes that lead to mucositis preclude the development and testing of effective treatment, despite the fact that the model of the disease outlined above permits an experimental approach for elucidating its biological mechanisms, including the molecular and cellular pathways leading to inflammation, disease and injury of the cells and tissues in the mucosa (6-8).

In oncology patients, management of mucositis may require, in some cases, a 1 week interruption of cancer therapy. Progress in the prevention and management of mucositis will improve quality of life, reduce cost of care, and facilitate completion of more intensive cancer chemotherapy and radiotherapy protocols. Improved management of mucositis will also allow implementation of cancer treatment protocols that are currently excessively mucotoxic but may produce higher cure rates. In these patients, the severity of mucositis depends upon the aggressive nature of the cancer therapy, the patient's state of immune suppression, the patient's white blood cell count, and the patient's general oral health and hygiene. Whether or not smoking and drinking behaviors may contribute to mucositis is still controversial (9-11). When local impairment of immune surveillance and consequential infection are not present, mucositis can heal by itself (12).

A short course of systemic prednisone (40-80 mg daily for not more than 1 week) is often recommended, and has been helpful in reducing inflammation and discomfort. Bioactive/ growth factors, hormones or interleukins have also been found to be effective for modifying epithelial metabolism and reducing the susceptibility of the tract to mucositis. Protective mucosal coatings such as sucralfate, alone or in combination with antibiotics and analgesics, help reduce mucositis and its associated pain and discomfort. Anti-inflammatory drugs, such as triclosan and indometacin reduce the duration and severity of mucositis in some cases and topical applications of vitamin $\mathrm{A}$ and $\mathrm{E}$ also have been used in attempts to reduce mucositis. The use of low-energy laser therapy can reduce the incidence of mucositis and enhance epithelial healing (7).

Recent developments in evidence-based medical care (13) permit a stringent evaluation of the literature. It must be emphasized that evidence-based research (EBR) in medicine rests on systematic reviews. This is quite distinct and different from medicine based on the evidence, which pertains to the traditional approach of modern Western medicine that relies on a few (or single) published communications. EBR in medicine refers to the practice of medicine grounded on the consensus statement obtained from the systematic research on all available published reports.

Results of one systematic review indicate that ice chips (odds ratio, $\mathrm{OR}=0.42 ; 95 \%$ confidence interval $(\mathrm{CI})=$ $0.19-0.93$ ) and benzydamine have the strongest scientific evidence of support for prophylaxis of mucositis (14). In 
point of fact, the literature supports use of benzydamine for prophylaxis of mucositis caused by conventional fractionated head and neck radiotherapy, and cryotherapy for short halflife stomatoxic chemotherapy, such as bolus fluorouracil (3). A Cochrane-sponsored systematic review has also shown that several interventions have some benefit at preventing or reducing the severity of mucositis associated with cancer treatment. The data indicate that these benefits are specific for certain cancer types and treatment. The findings established, moreover, that the number needed to treat (NNT) to prevent one patient experiencing mucositis over a baseline incidence of $60 \%$ for amifostine is $\mathrm{NNT}=33\left(\mathrm{CI}^{95}=\right.$ $20-100)$, for antibiotic paste or pastille NNT $=13\left(\mathrm{CI}^{95}=\right.$ $8-50)$ and for ice chips NNT $=5\left(\mathrm{CI}^{95}=2-31\right)(11)$.

Whereas the precise immune components involved in the etiology of mucositis are unclear, EBR has established that applications of granulocyte-macrophage-colony stimulating factor (GM-CSF) prevent the onset or the exacerbation of oropharyngeal mucositis (risk ratio, $\mathrm{RR}=0.51$, $\left.\mathrm{CI}^{95}=0.29-0.91 ; \quad \mathrm{NNT}=3, \mathrm{CI}^{95}=2-20\right)$ (11). Clinical trials suggest that GM-CSF has clinical benefits beyond enhancing neutrophil recovery, including shortening the duration of mucositis and diarrhea (15), stimulating dendritic cells, preventing infection, acting as an adjuvant vaccine agent and facilitating antitumor activity (16).

The colony-stimulating factors (CSFs) are a family of cytokines central to the development and maturation of cells of the immune system, the modulation of their functional responses, as well as the maintenance of immune homeostasis and overall immunity. This group of glycoproteins consists of the macrophage-CSF (M-CSF), granulocyte-CSF (G-CSF), GM-CSF and multi-CSF [interleukin(IL)-3]. GM-CSF functions at early stages of lineage commitment regulating the expansion and maturation of hematopoietic progenitor cells (17). GM-CSF is a proinflammatory cytokine that stimulates proliferation and differentiation of neutrophilic, eosinophilic and monocytic lineages of cellular immunity. In immunosuppressed patients, and in murine models of therapeutic immune suppression, GM-CSF administration is effective in boosting the innate immune response, while continuing to suppress the adaptive immune response to prevent graft rejection (18).

\section{Molecular Immunology}

Molecular cartography, the science of recognizing and identifying the multifaceted and intricate array of interacting genes and gene products that characterize the function and specialization of each individual cell in the context of cell-cell interaction, tissue and organ function, and system's biology in general, is among the most promising and cutting-edge trends in the health sciences today. DNA sequencing technology of the 1970s and the 1980s gave way to genomics, the characterization of proteomics, and the validation of the interactome, the map of all possible interactions among genes and gene products in the organism in response to stimuli from within (e.g. hormonal response to challenges, viral infection), and from challenges that come from outside the organism (e.g. alcohol use and abuse). The analysis of gene expression in tissues, cells and biologic systems by microarray allows discovery-based research to characterize either new genes with unknown function or genes not previously known to be involved in a biologic process (19). Integrating interactome maps with systematic genetic perturbations will be useful for developing a systems biology approach to this and other signaling modules (20).

The relevance of molecular cartography in immunology pertains to every domain of physiology, including immunity. Molecular cartography provides the fundamental knowledge and understanding of the genomic, the proteomic and interactomic processes that regulate the emergence, stability and function of immune cell populations, and the mechanisms by which new populations arise following antigenic triggers and immune activation. Molecular cartography of immunity also produces new fundamental knowledge with respect to the processes that determine the functional response of immune cells, such as, for instance, the ability of immune $\mathrm{T}$ cells to produce cytokines or to migrate across an endothelial barrier toward an antigen (5).

The value of molecular cartography in immunology lies not only in the characterization of the fundamental biologic mechanisms that control and regulate immune processes and events in the healthy individual, but as well, and perhaps more importantly, in revealing the modes and modalities by which the organism adapts to physiological insults and stressful challenges, injury (e.g. the injurious sequelae of smoking or alcohol) and disease (e.g. cancer).

Molecular cartography also plays a critical role in the elucidation of pathological processes, including mucositis, and in the development of novel or improved modes of treatment interventions. Careful study of the genome, proteome and interactome provides essential new knowledge about the process of disease. This is one of the most attractive features of molecular cartography for pharmaceutical companies, which labor to develop new vaccine protocols and new therapeutic drugs. Molecular cartography, or 'meta-genomics' as it is often labeled in the context of the drug development industry, is promising, but it is also fraught with problems and caveats due in part to our limited knowledge and understanding, at present, of the gargantuan nature of these systems, due to the emerging realization that genes and their protein products have the potential of interacting in a multitude of ways, and because drugs targeted to specific molecular pathways have the potential of producing troubling side effects.

Case in point is the role of GM-CSF in the SOCS pathway, which may underlie the pathobiological process of mucositis. The activation of the family of suppressors of cytokine signaling (SOCS) has direct effects on transmembrane and transcytosolic signaling pathways that are central to immune cell activation, and that involve the janus kinase/signal transducer and activator of transcription (JAK/STAT) and the Ras pathways (5). The activation of these pathways is essential for dendritic cell differentiation and maturation. STAT6 signaling 
pathway, for instance, is constitutively activated in immature dendritic populations and declines as these cells mature. This sequence of molecular events is associated with a marked induction of SOCS1, SOCS2, SOCS3, as well as cytokineinduced Src homology 2-containing protein expression. STAT1 signaling is not altered during the maturation process of dendritic cells, remains unaffected by SOCS, but is optimally induced by IL-4 and GM-CSF. IL-4, in turns, specifically activates SOCS-1, whereas GM-CSF activates SOCS-3 during the maturation of dendritic cells (21).

AkT [phosphoinositide 3-kinase, also known as protein kinase B (PKB)] and the tumor suppressor, PTEN (phosphatase and tensin homolog deleted on chromosome 10, which removes phosphates primarily from lipids), also play an important role in modulating signaling events, including Ras, and functional cellular immune responses, including activation and migration. The other important pathway related to SOCS is the P3 member of the first forkhead-box (FOX) transcription factor (FoxP3). FoxP3 functions as a transcriptional repressor, targeting composite NF-AT/AP-1 sites in cytokine gene promoters. The region responsible for NF-AT inhibition has been mapped to the amino terminus of FoxP3. Molecular experiments show that introduction of FoxP3 into conventional mouse $\mathrm{T}$ cells converts these cells to the regulatory $\mathrm{T}$ cell (Treg) phenotype $\left(\mathrm{CD} 4^{+} \mathrm{CD} 25^{+}\right) . \mathrm{CD} 4^{+} \mathrm{CD} 25^{-}$cells can be converted to the $\mathrm{CD} 25^{+}$Treg phenotype following in vitro stimulation, with associated acquisition of Treg-like function, and induction of FoxP3 expression (22-24). Proteins that interact with nucleophosmin-anaplastic lymphoma kinase (NPM-ALK) tyrosine kinase also play important roles in mediating downstream cellular signals. These, identified by microarray include, PI3-K, JAK2, JAK3, STAT3, Grb2, IRS, and PLCgamma1, Rho-GTPase activating protein (RAB35), kinases (MEK kinase 1 and 4, PKC, MLCK, cyclin G-associated kinase, EphA1, JNK kinase, MAP kinase 1), phosphatases (meprin, PTPK, protein phosphatase 2 subunit), and heat shock proteins (Hsp60 precursor), as well as SOCS (25).

CD28 ligation increases the proliferative response of both $\mathrm{CD}^{+}{ }^{+}$and $\mathrm{CD}^{+}{ }^{+} \mathrm{T}$ cell subsets via these molecular pathways. The response of the $\mathrm{CD}^{+}$subset is more marked in terms, particularly, of 5-fold increase in GM-CSF, compared with a 2 -fold increase in this cytokine by $\mathrm{CD} 8^{+} \mathrm{T}$ cells. The naive $\mathrm{CD}^{+}$subset $\left(\mathrm{CD} 45 \mathrm{RA}^{+}, \mathrm{CD}_{4} 4^{-}\right)$is the most CD28-responsive lymphocyte population to CD28-mediated costimulation (26). $\mathrm{CD} 25^{+} \mathrm{CD} 4^{+}$Tregs are required for the maintenance of peripheral tolerance to certain self antigens. The suppressive function of Tregs is critically dependent on immature myeliod dendritic cells and is reversed by the maturation of this cell population induced by GM-CSF (27).

The GM-CSF/IL-3/IL-5 receptors are a family of heterodimeric transmembrane proteins expressed by dendritic cells and other myeloid lineage cells, which also transmit activation signals through these complex pathways. Each receptor has a unique ligand-binding $\alpha$ chain, but they share a common $\beta c$ chain. Binding of GM-CSF activates JAK2, which rapidly induces tyrosine phosphorylation of the $\beta \mathrm{c}$ chain, but not the $\alpha$ chain. Molecular mutation experiments, however, have established that $\beta \mathrm{c}$ chain tyrosine residues are not necessary for activation of the JAK/STAT pathway, or for proliferation, viability, or adhesion signaling, although they significantly increase the magnitude of these responses (28). That GM-CSF and its receptor are expressed in the brain, and appear to play an important role in modulating various peripheral and central nervous system functions (29) suggests an important neuroimmune role of GM-CSF in the etiology of mucositis, which could be exploited for novel modes of treatment intervention.

In this context, it is possible and even probable that research will uncover a neuronal receptor/ligand interaction (e.g. GM-CSF) with Treg cells that under stress may inhibit the inhibitory action of the FoxP3 interaction with NF-AT/AP-1 sites in cytokine promoters. Indeed, lymphocytes express several neuron-like (e.g. $\beta$-adrenergic) and hormonal receptors (e.g. glucocorticoids, ACTH, opioid), whose transmembrane signaling could alter FoxP3, and SOCS and the associated signaling network.

In closing, and recalling the recent findings of the vagal efferents on immune suppression (30), psychosocial stress, which is an important etiological factor for mucositis, could soon be shown to alter the expression of the acetylcholinesterase gene. This gene product is now known to present in at least three alternative proteins that are implicated in a wide variety of regulatory mind-body responses. Stressinduced alternative gene splicing such as this could become a major mind-body pathway of psychosocial genomics, particularly in terms of the immunopathology of mucositis, in the coming decade (31).

We have established in previous studies a direct link between the parasympathetic glossopharyngeal innervation (CN IX) and immune surveillance of the oropharyngeal mucosa (32). We predict that future genomic research will uncover the fundamental signaling pathways that are involved in the pathological processes of mucositis: keratinocyte death, which lead to suppression of immune surveillance, and that result in bacterial and fungal invasion of the stoma. Together these advances will lead to improved traditional, complementary and alternative treatment for patients with mucositis.

\section{Implications for Interventions in Complementary and Alternative Medicine (CAM)}

The purpose of this paper was not meant to explore and discuss in an exhaustive fashion the several excellent papers written in the domain of mucositis, which would be akin to generating a traditional narrative review. Rather, we sought to cross the new frontier of the evidence-based discourse, and to focus on what is known and available in the systematically reviewed body of evidence: in EBR in mucositis. We examined the implications of EBR for the development of CAM-based interventions, with the focal point of interest being not every and all pathological 
processes involved in mucositis, but specifically molecular immunity. The one treatment for mucositis that rests upon molecular immune pathways of this disease, and that has been examined by means of systematic EBR is GM-CSF-based intervention.

Immediate relevance and applications of the findings we discuss in this paper will pertain to the clinical treatment of mucositis. This is critical since in 2004 alone, close to 1.5 million Americans were diagnosed with cancer and received some combination therapies for this disease, including surgery, radiation, chemotherapy, immunotherapy and/or cell transplantation. A range of side effects accompanies these different types of cancer therapy, from the mild and transient alopecia, nausea and neutropenia, to the chronic signs of fatigue and lymphedema, to the late and potentially life threatening cardiomyopathy, and to severe oral manifestations. Mucositis, the principal oral complication from cancer therapies is common and can substantially impair the comfort and function of patients during and after the treatment protocol, and the patient's willingness to adhere to or complete the prescribed therapy (7).

The clinical significance of mucositis is heightened by the fact that not only individuals undergoing cancer therapy are at risk but also immunosuppressed patients in general, including subjects who are seropositive for the immunodeficiency virus-1 (HIV) with or without full-blown acquired immune deficiency syndrome (AIDS) $(33,34)$. The elderly as well are at risk, and older subjects have increased sensitivity to oral toxicity, in particular, mucositis (35).

EBR in complementary and alternative interventions for mucositis has established that prophylactic administration of scavenger or anti-inflammatory agents can block or reverse the etiology of this condition. Mouthwashes and gargling agents may be beneficial complementary interventions for patients with oral mucositis, in that these treatments may shorten the duration and severity of symptoms. The regular use of mouthwashes, mouth coatings, antibiotics and analgesics has been shown to be essential, before and during loss and ablation of the epithelial layer. Glutamine supplements seem to be beneficial in the repair and recovery phase (7).

Few controlled clinical trials of psychological interventions for pain relief exist in spite of frequent support for their importance as adjuncts to medical treatment, particularly in the context of mucositis-associated distress. In a study of pain associated with oral mucositis, cancer patients receiving bone marrow transplants were administered therapist support, relaxation and imagery training, cognitive-behavioral coping skills with relaxation and imagery, or placebo control. Patients who received either relaxation and imagery alone, the package of cognitive-behavioral coping skills with added relaxation and imagery reported significantly less pain, compared with patients in the other groups. Cognitive-behavioral skills were not more beneficial than relaxation and imagery alone $(P=0.103)$, suggesting relaxation and imagery training, whether by itself or in conjunction with cognitive skills reduces cancer treatment-related mucositis. Training patients in cognitive-behavioral skills in addition to relaxation with imagery failed to further improve outcomes (36). A systematic review conducted following the stringent protocol and recommendations of EBR in medicine further confirmed the value of relaxation and imagery as a complementary medicine intervention in oral mucositis in terminal cancer patients under radiation or chemotherapy treatment (37).

Alternative interventions to Western pharmaceutical treatment involve traditional Asian herbal medicine. For instance, the Japanese herb Syousaikotou was tested as a gargling agent for patients receiving chemotherapy, as a proactive measure against the onset of mucositis. Compared with the Placebo group that used a mouthwash of providone-iodine and amphotericin B, the Syousaikotou gargle group showed a significantly decreased incidence of stomatitis, and significantly reduced oral mucosal irritation, inflammation and pain. Side effects of Syousaikotou treatment were limited to $<10 \%$ of the patients treated, who complained of halitosis and of oil and grass smells (38). In related study, oral administration of the putative antioxidant oren-gedoku-to (Japanese, Coptidis rhizoma; Huanglin-Jie-Du-Tang in Chinese pharmacopoeia) reduced significantly incidence of mucositis and of stomatitis (27.9\%), compared with $71.6 \%$ in those who received a gargle consisting of the placebo group treated with allopurinol, sodium gualenate and povidone-iodine $(71.6 \%)$. Herbal treatment also significantly decreased anticancer interventioninduced diarrhea (9.3\%), compared with the placebo group (31.7\%) (39). Evidence-based systematic reviews of the research evidence on the effectiveness and efficacy of herbal medicine in the treatment of mucositis are lacking to date.

Taken together and despite current advances in knowledge and fundamental molecular understanding of the etiology and the immunopathology of mucositis, it is clear from current EBR that the limited number of available clinical trials precludes the recommendation of one over another treatment intervention for mucositis. Future advances will depend upon well-designed and well-conducted studies with sufficient numbers of participants to perform subgroup analyses by type of disease $(2,11)$.

In conclusion, this paper discussed three main domains of mucositis. It began by defining it, then discussed what is known about treatments, and examined the fundamental molecular immunology of mucositis. This discussion, within the cadre of evidence-based medicine and CAM, led to an evaluation of the relevance to evidence-based CAM, the focal point of the paper, its raison d'être in light of immediate potential relevance and applications to the clinical treatment of mucositis.

\section{References}

1. Pico JL, Avila-Garavito A, Naccache P. Mucositis: its occurrence, consequences, and treatment in the oncology setting. Oncologist 1998;3: 446-51.

2. Rubenstein EB, Peterson DE, Schubert M, Keefe D, McGuire D, Epstein J, et al. Clinical practice guidelines for the prevention and treatment of cancer therapy-induced oral and gastrointestinal mucositis. Cancer 2004; 100:2026-46. 
3. Epstein JB, Schubert MM. Oropharyngeal mucositis in cancer therapy. Review of pathogenesis, diagnosis, and management. Oncology 2003;17: 1767-79, discussion 1779-82, 1791-2.

4. Sonis ST. A biological approach to mucositis. J Support Oncol 2004;2: 21-32; discussion 35-6.

5. Chiappelli F. Immunity. In: Fink G (ed). Encyclopaedia of Stress. Academic Press, New York, 2005 (in press).

6. Alvarado Y, Bellm LA, Giles FJ. Oral mucositis: time for more studies. Hematology 2002;7:281-9.

7. Duncan M, Grant G. Oral and intestinal mucositis-causes and possible treatments. Aliment Pharmacol Ther 2003;18:853-74.

8. Kwong KK. Prevention and treatment of oropharyngeal mucositis following cancer therapy: are there new approaches?. Cancer Nurs 2004;27: 183-205.

9. Dodd MJ, Miaskowski C, Shiba GH, Dibble SL, Greenspan D, MacPhail L, et al. Risk factors for chemotherapy-induced oral mucositis: dental appliances, oral hygiene, previous oral lesions, and history of smoking. Cancer Invest 1999;17:278-8.

10. Bolwell BJ, Kalaycio M, Sobecks R, Andresen S, Kuczkowski E, Bernhard L, et al. A multivariable analysis of factors influencing mucositis after autologous progenitor cell transplantation. Bone Marrow Transplant 2002;30:587-91.

11. Clarkson JE, Worthington HV, Eden OB. Interventions for preventing oral mucositis for patients receiving treatment. Cochrane Database Syst Rev 2003;3:CD000978.

12. Loprinzi CL, Gastineau DA, Foote RL. Oral complications. In: Abeloff MD, Armitage JO, Lichter AS, Niederhuber JE (eds). Clinical Oncology. New York: Churchill Livingstone, 1995, 741-54.

13. Blocklehurst P, McGuire W. Evidence-based care. Br Med J 2005;330: 36-8.

14. Scully C, Epstein J, Sonis S. Oral mucositis: a challenging complication of radiotherapy, chemotherapy, and radiochemotherapy: part 1, pathogenesis and prophylaxis of mucositis. Head Neck 2003;25:1057-70.

15. Nemunaitis J, Rosenfeld CS, Ash R, Freedman MH, Deeg HJ, Appelbaum F, et al. Phase III randomized, double-blind placebocontrolled trial of rhGM-CSF following allogeneic bone marrow transplantation. Bone Marrow Transplant 1995;15:949-54.

16. Buchsel PC, Forgey A, Grape FB, Hamann SS. Granulocyte macrophage colony-stimulating factor: current practice and novel approaches. Clin J Oncol Nurs 2002;6:198-205.

17. Barreda DR, Hanington PC, Belosevic M. Regulation of myeloid development and function by colony stimulating factors. Dev Comp Immunol 2004;28:509-54.

18. Xu J, Lucas R, Wendel A. The potential of GM-CSF to improve resistance against infections in organ transplantation. Trends Pharmacol Sci 2004;25:254-8.

19. van der Pouw Kraan TC, Kasperkovitz PV, Verbeet N, Verweij CL. Genomics in the immune system. Clin Immunol 2004;111:175-85.

20. Tewari M, Hu PJ, Ahn JS, Ayivi-Guedehoussou N, Vidalain PO, Li S, et al. Systematic interactome mapping and genetic perturbation analysis of a C. elegans TGF-beta signaling network. Mol Cell 2004;13:469-82.

21. Jackson SH, Yu CR, Mahdi RM, Ebong S, Egwuagu CE. Dendritic cell maturation requires STAT1 and is under feedback regulation by suppressors of cytokine signaling. J Immunol 2004;172: 2307-15.
22. Coffer PJ, Burgering BM. Forkhead-box transcription factors and their role in the immune system. Nat Rev Immunol 2004;4:889-99.

23. Thompson C, Powrie F. Regulatory T cells. Curr Opin Pharmacol 2004;4: 408-14.

24. Kim D, Dan HC, Park S, Yang L, Liu Q, Kaneko S, et al. AKT/PKB signaling mechanisms in cancer and chemoresistance. Front Biosci 2005; $10: 975-84$

25. Crockett DK, Lin Z, Elenitoba-Johnson KS, Lim MS. Identification of NPM-ALK interacting proteins by tandem mass spectrometry. Oncogene 2004;23:2617-29.

26. Rochford R, Riggs JE, Clavo A, Ernst DN, Hobbs MV. Differential effects of CD28 costimulation upon cytokine production by CD4+ and CD8+ T cells. Immunobiology 2004;209:513-22.

27. Kubo T, Hatton RD, Oliver J, Liu X, Elson CO, Weaver CT. Regulatory T cell suppression and anergy are differentially regulated by proinflammatory cytokines produced by TLR-activated dendritic cells. J Immunol 2004;173:7249-58.

28. Okuda K, Foster R, Griffin JD. Signaling domains of the beta c chain of the GM-CSF/IL-3/IL-5 receptor. Ann NY Acad Sci 1999;872:305-12; discussion 312-3.

29. Franzen R, Bouhy D, Schoenen J. Nervous system injury: focus on the inflammatory cytokine 'granulocyte-macrophage colony stimulating factor'. Neurosci Lett 2004;361:76-8.

30. Czura CJ, Friedman SG, Tracey KJ. Neural inhibition of inflammation: the cholinergic anti-inflammatory pathway. J Endotoxin Res 2003;9: 409-13.

31. Rossi EL. Stress-induced alternative gene splicing in mind-body medicine. Adv Mind Body Med 2004;20:12-9.

32. Romeo H, Tio DL, Rahman SU, Chiappelli F, Taylor AN. The glossopharyngeal nerve as a novel pathway in immune-to-brain communication: Relevance to neuroimmune surveillance of the oral cavity. J Neuroimmunol 2001;115:91-100.

33. Fischbein NJ, Dillon WP. Imaging of otolaryngologic manifestations of HIV infection. Neuroimaging Clin N Am 1997;7:375-99.

34. Handforth J, Sharland M. Triple nucleoside reverse transcriptase inhibitor therapy in children. Paediatr Drugs 2004;6:147-59.

35. Lichtman SM. Physiological aspects of aging. Implications for the treatment of cancer. Drugs Aging 1995;7:212-25.

36. Syrjala KL, Donaldson GW, Davis MW, Kippes ME, Carr JE. Relaxation and imagery and cognitive-behavioral training reduce pain during cancer treatment: a controlled clinical trial. Pain 1995;63: 189-98.

37. Pan CX, Morrison RS, Ness J, Fugh-Berman A, Leipzig RM. Complementary and alternative medicine in the management of pain, dyspnea, and nausea and vomiting near the end of life. A systematic review. J Pain Symptom Manage 2000;20:374-87.

38. Matsuoka H, Mizushima Y, Kawano M, Tachibana N, Sawada Y, Kato S, et al. Clinical availability of the herbal medicine, Syousaikotou, as a gargling agent for prevention and treatment of chemotherapy-induced stomatitis. Gan To Kagaku Ryoho 2004;31:2017-20.

39. Yuki F, Kawaguchi T, Hazemoto K, Asou N. Preventive effects of oren-gedoku-to on mucositis caused by anticancer agents in patients with acute leukemia. Gan To Kagaku Ryoho 2003;30:1303-7.

Received January 3, 2005; accepted September 15, 2005 


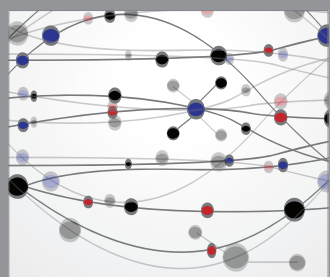

The Scientific World Journal
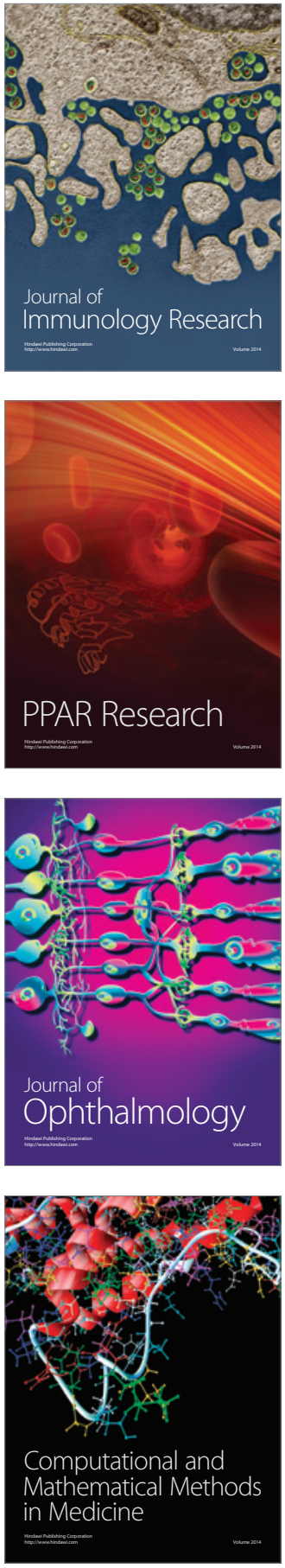

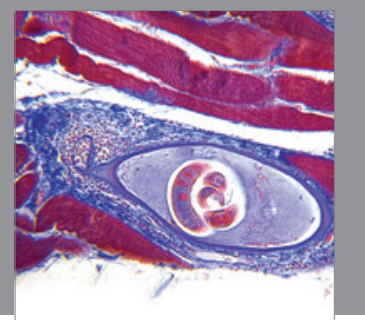

Gastroenterology

Research and Practice
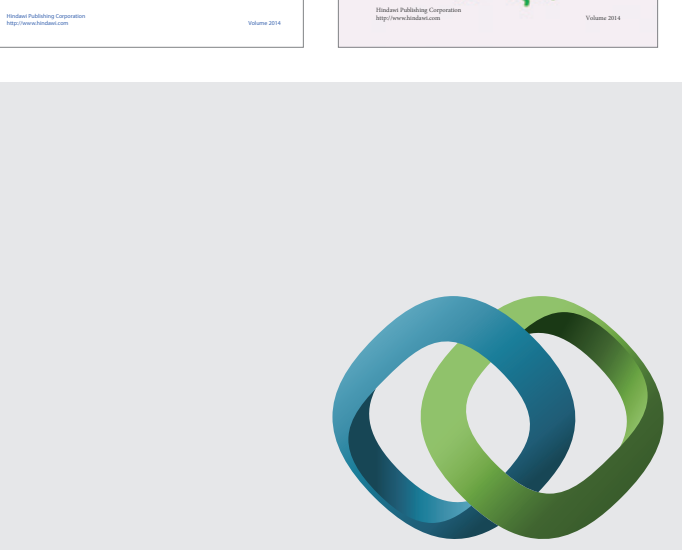

\section{Hindawi}

Submit your manuscripts at

http://www.hindawi.com
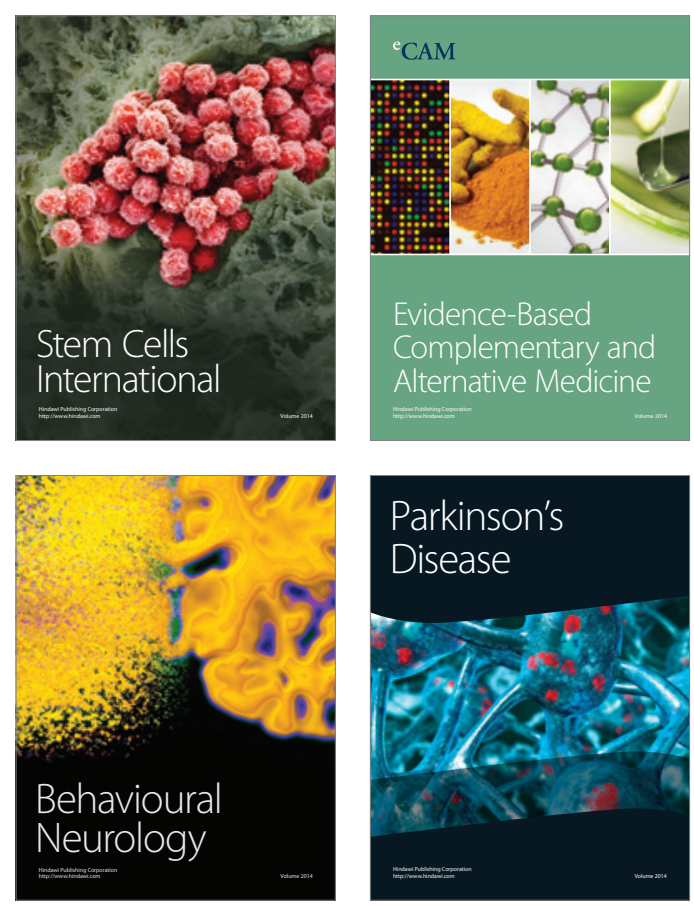

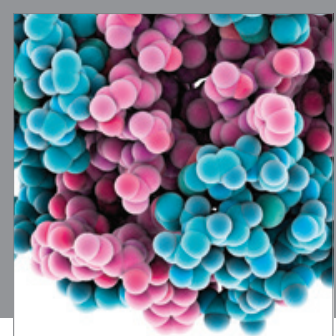

Journal of
Diabetes Research

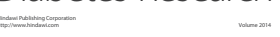

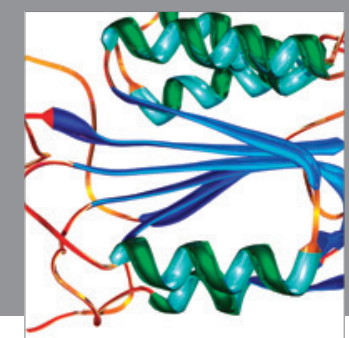

Disease Markers
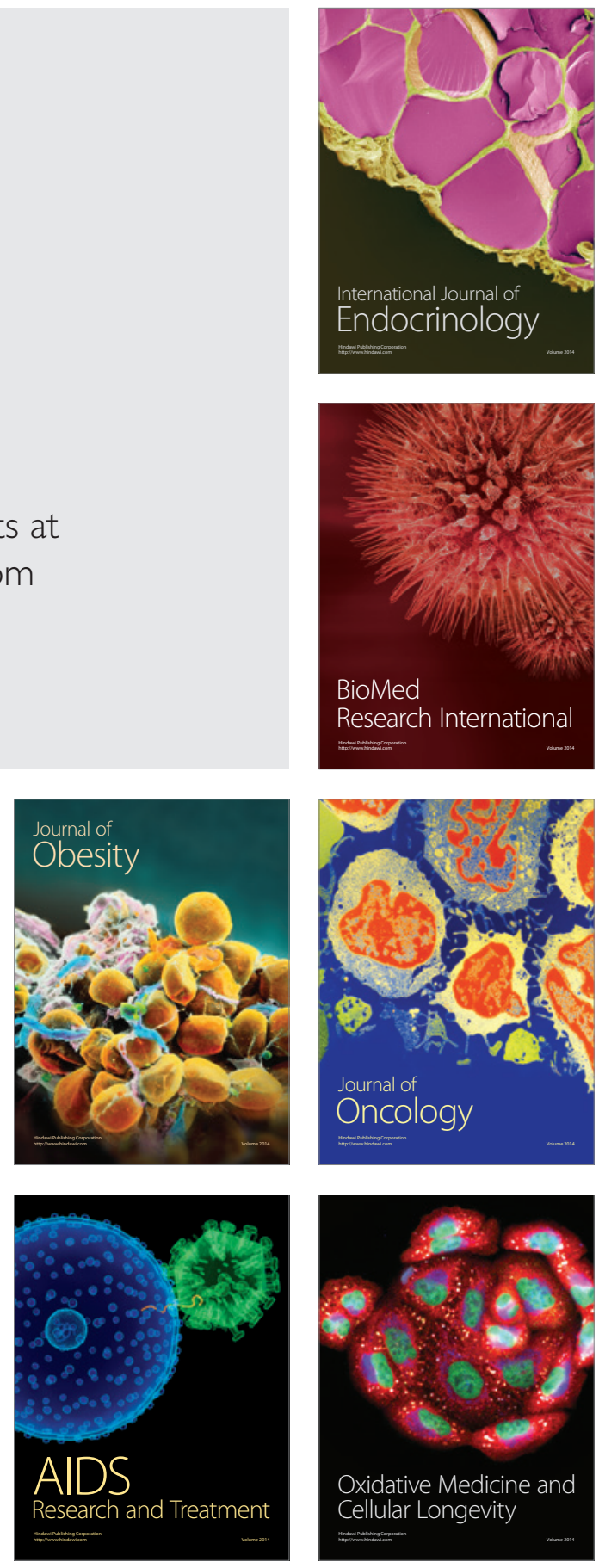